\title{
Trajectories of Cognitive Function and Their Machine Learning-based Prediction Models in Older Adults: a 16 Years Follow-up in the Chinese Longitudinal Healthy Longevity Survey
}

\section{Yafei Wu}

Xiamen University School of Public Health

Maoni Jia

Xiamen University School of Public Health

Chaoyi Xiang

Xiamen University School of Public Health

Shaowu Lin

Xiamen University School of Public Health

\section{Zhongquan Jiang}

Xiamen University School of Public Health

Ya Fang ( $\nabla$ fangya@xmu.edu.cn )

Xiamen University School of Public Health https://orcid.org/0000-0002-9895-3234

\section{Research}

Keywords: Cognitive trajectories, Machine learning, Growth mixture model, MMSE

Posted Date: July 26th, 2021

DOl: https://doi.org/10.21203/rs.3.rs-725498/v1

License: (c) (i) This work is licensed under a Creative Commons Attribution 4.0 International License.

Read Full License 


\section{Abstract}

Background: Cognitive decline shows heterogeneous with increasing age. The trajectory of cognitive function is yet to be fully investigated in older Chinese. Early identification of such trend is essential for the prevention of high-risk population related to cognitive decline. This study aimed to explore the heterogeneity and determinants of cognitive trajectories, and construct prediction models for distinguishing cognitive trajectories among the elderly Chinese at a community level.

Methods: This study included 3502 older adults aged 65-105 years at their first observations in the Chinese Longitudinal Healthy Longevity Survey from 2002 to 2018. The Chinese version Mini-Mental State Examination (MMSE) was used for measuring cognitive function. The heterogeneity of cognitive trajectories was identified through mixed growth model, and the determinants of cognitive trajectories were analyzed by logistic regression. Under a consideration of feature selection, machine learning algorithms, namely logistic regression (LR), support vector machine (SVM), and an integrated algorithm combining LR and SVM (stacking), were used to predict cognitive trajectories using epidemiological variables. Area under the receiver operating characteristic curve (AUROC) and brier score were used to assess discrimination and calibration, respectively.

Results: Two distinct cognitive trajectories were identified according to the changes of MMSE scores: stable-function (93.6\%), and rapid-decline (6.4\%). Older age, female gender, Han ethnicity, having no schooling, rural residents, low frequency leisure activities, and low baseline ADL score were associated with the rapid decline in cognitive function. For classification of two trajectories, the performance of LR, SVM and stacking algorithms with feature selection were comparable, of which achieved 0.66 of balanced accuracy, 0.74 of sensitivity, 0.73 of F1 score, 0.66 of AUROC, and 0.25 of brier score. Among the predictors, age and psychological well-being were the most effective variables for distinguishing two trajectories.

Conclusions: Two cognitive trajectories were identified among older Chinese. The identified determinants of trajectories could be targeted for constructing early risk prediction models.

\section{Background}

Dementia is a degenerative disease of the central nervous system accompanied by cognitive decline. According to the World Alzheimer's Report, more than 50 million people worldwide were estimated to be living with dementia in 2020. Moreover, the number will increase to 152 million by 2050 with rapid aging [1]. Until now, the etiology and pathogenesis of dementia are still unclear, and there is no effective cure in clinical practice [2]. About three quarters of dementia patients worldwide were not diagnosed in time, especially in low- and middle-income countries. It was estimated that the annual global losses related to dementia were $\$ 818$ billion, which was equivalent to the output of the 18th largest economy [1].

Therefore, it is crucial to identify the high-risk individuals of cognitive decline and evaluate their long-term changing patterns for the timely prevention of dementia. 
Cognitive function is constantly changing with age increasing. Previous studies have demonstrated that heterogeneity exists in trajectories of cognitive function over time [3-6]. While trajectory of cognitive function is yet to be fully investigated in older Chinese. Meanwhile, previous prediction studies related to cognitive function mainly focused on diagnostic conversion, especially for mild cognitive impairment $(\mathrm{MCl})$ converts into Alzheimer disease (AD) [7-10], and according to our understanding, only few studies started to make predictions of cognitive trajectories [11, 12]. Therefore, identification and prediction of trajectories of cognitive function have significant implications for primary prevention targeted at potential vulnerable older adults.

Machine learning $(\mathrm{ML})$, which is a set of computational methods that can discover complex nonlinear relationships between inputs and outputs, has been widely used in disease prediction [13]. In medical practice, ML can process complex medical data and construct prediction models for decision-making. Most models have been derived using logistic regression or support vector machine in predicting cognitive-related diseases as well as other psychological diseases, and have showed decent performance $[14,15]$. Currently, with the development of computer technology and the accumulation of high-volume data, machine learning has been played an increasingly important role in health risk prediction.

This study focused on two issues: first, mixed growth model was used to explore the trajectories of cognitive function among the older Chinese, and the determinants of cognitive trajectories were also analyzed. Then, under a consideration of feature selection (recursive feature elimination), machine learning algorithms, namely logistic regression (LR), support vector machine (SVM), and an integrated method combining both LR and SVM (known as stacking), were used to predict the types of cognitive trajectories using epidemiological variables.

\section{Methods}

\section{Study design and participants}

This study retrospectively collected data from the Chinese Longitudinal Healthy Longevity Survey (CLHLS). CLHLS is an ongoing, prospective cohort study of community-dwelling Chinese older individuals $[16,17]$. It covers 22 of the 31 mainland provinces, encompassing $85 \%$ of the total population in China. Starting in 1998, a follow-up investigation was conducted every 3 years, and there are totally 8 waves $(1998,2000,2002,2005,2008,2011,2014$, and 2018) so far. The CLHLS study was approved by the Research Ethics Committee of Peking University (IRB00001052-13074), and was publicly available at the Peking University Open Research Data (https://opendata.pku.edu.cn/dataverse/CHADS). More details of the study design have been described in previous studies, and the survey data have been widely reported as high-quality [18].

The first 2 waves (1998 and 2000) were excluded because they mainly targeted for participants older than 80 years. Thus, six recent investigations from 2002 to 2018 were used for analysis. Data in 2002 were selected as baseline in this study. For trajectories analysis, those who had complete information in the Chinese version Mini-Mental State Examination (MMSE) for at least two waves were included. Finally, 
a total of 3502 participants aged $\geq 65$ years were included. The flow chart of the CLHLS follow-up and the sample selection of the current analysis is presented in Fig. 1.

\section{Cognitive Assessment}

The CLHLS used the Chinese version of the Mini-Mental State Examination (MMSE), whose validity and reliability have been verified $[17,19]$, as a measure of global cognitive function at each wave. MMSE contains a total of 24 questions, involving 7 dimensions of orientation, food counting within one minute, memory, calculation, drawing, recall and language. Except for food counting within one minute (one point for each food, and not exceed 7 points of a maximum score), other questions were coded as follows: 1 point (correct answer) and 0 point (wrong answer). The total scores of the MMSE range from 0 to 30 , with higher scores representing higher cognitive function. The MMSE scores were used to identify the potential trajectories of cognitive function in the current analysis.

\section{Measurement of Predictors}

Predictors in this study included sociodemographic characteristics, lifestyles, psychological well-being (PWB) and physical activity, and chronic diseases. For sociodemographic characteristics, age, sex (man, woman), ethnicity (Han ancestry, minority), education (illiterate, primary school, junior high and above), marital status (unmarried, separated or divorced or widowed, married), residence (rural, urban), and coresidence (yes, no) were included. For lifestyles, regular fruits intake (yes, no), regular vegetables intake (yes, no), regular tea consumption (yes, no), smoker (yes, no), alcohol drinker (yes, no), regular exercise (yes, no), and leisure activity (yes, no) were considered. Specifically, leisure activities included housework, personal outdoor activities, garden work, reading newspapers or books, raising domestic animals or pets, playing cards or mahjong, watching TV or listening to the radio, and taking part in some social activities. According to previous study [5], each item had 5 levels and was coded as follows: "almost every day" (coded as 1), "not daily, but once a week" (coded as 2), "not weekly, but at least once a month" (coded as 3), "not monthly, but sometimes" (coded as 4), and "never" (coded as 5). The total scores of the above 8 items ranged 8-40. A high frequency was defined as "being scored at 40th percentile or below", and a low frequency as “being scored over 40th percentile”. From 1998 to 2005 in CLHLS, 7 items (optimism, conscientiousness, personal control, happiness, neuroticism, loneliness, and self-esteem) were used to test the psychological state. The use of these items for measuring PWB has been used in several previous studies [20-22]. Specifically, if the item is helpful to PWB, we coded as follows: 5 ("always"), 4 ("often"), 3 ("sometimes"), 2 ("seldom"), and 1 ("never"); and if the item is harmful to PWB, we coded in an opposite way, and 0 point were code for "unable to answer" for all items. Therefore, the total scores of PWB ranged $0-35$, of which a higher score indicating much better psychological state. Physical activity included activities of daily living (ADL) and instrumental ADL (IADL). ADL was assessed by the 6 daily activities (bathing, dressing, continence, using the toilet, indoor transferring, and feeding themselves), and IADL was assessed by the 8 instrumental activities (shopping, cooking, visiting neighbors, doing laundry, walking continuously for $1 \mathrm{~km}$, continuously crouching and standing up 3 times, lifting a weight of $5 \mathrm{~kg}$, and taking public transportation) [23]. Each item was coded as: 0 ("with complete assistance"), 1 ("with part assistance"), or 2 ("independently"). So, the total scores of ADL and IADL ranged 0-12, and 0-16, 
respectively. For chronic diseases, self-reported diagnosis of hypertension (yes, no), diabetes (yes, no), and stroke (yes, no) were selected. Detailed measurements of variables were summarized in Supplementary Table S1. The multiple imputation approach was applied to reduce the influence of missing values on predictors in the analyses.

\section{Feature selection}

Feature selection was performed for reducing the dimensions of variables and improving model performance. In the current study, recursive feature elimination (RFE) was used. RFE is an ML method for feature selection that combines with several classifiers to eliminate redundant variables, thus identifying the most important factors for each classifier [24]. In order to select the best combination of predictors, a 10-flod cross validation was combined with RFE, that was, RFE was performed on each subset of input data, and validation error of all subsets was calculated. Finally, the subset with smallest error was selected as the optimal combination.

\section{Trajectories of cognitive function}

The Growth Mixed Model (GMM) was used to explore the heterogeneity of cognitive trajectories, which could divide populations into several groups based on the differences in growth trajectories. Previous studies suggested that a latent growth curve model (LGCM) and latent class growth model (LCGM) should be used to explore the shape of growth curve and the number of potential trajectory classes before GMM analysis $[25,26]$. When the optimal LCGM model was selected, the GMM model was fitted subsequently. For model selection, statistical indices and interpretability are often considered. Statistical indices include sample-size adjusted Bayesian information criteria (SABIC), entropy, Vuong-Lo-MendellRubin likelihood ratio test (VLMR-LRT), and proportion of the smallest class. SABIC is an information criterion with a more reduction, representing an improvement of model. Entropy is a measure of classification accuracy, ranging from 0 to 1 . The larger the entropy, the better the trajectories classification. VLMR-LRT compares the results of the $k-1$ class model with $k$ class model. A significant $p$ value $(<0.05)$ indicates that $k$ class model is better than $k-1$ class model. Besides, each trajectory class must contain enough samples, no less than $5 \%$ of total population. Followed with previous studies [5, 23], trajectory classes ranged 1 to 5 were tested in this study, and then selected the most favorable class according to the above indices.

\section{Derivation and evaluation of cognitive trajectories prediction models}

For prediction of cognitive trajectories, the commonly used logistic regression (LR) and support vector machine (SVM) in the field of psychology were selected. Considering that LR and SVM are all single classifiers, so a new method that combines both LR and SVM (known as stacking) is further constructed for distinguishing different trajectories. Stacking is one of the ensemble learning algorithms, which can integrate various of $M L$ algorithms into achieving a more powerful learner [27]. For LR, two hyperparameters (types of penalty: "none", "L1", "L2", "elasticnet", and the regularization term $C$. searching from $0.01,0.1,1,10,100$ ) were tuned with 10-fold cross validation [24] in the training set, and 
finally penalty and C were set as "L1" and 10, respectively. For SVM, the kerne/ parameter ("linear", "rbf", "poly", "sigmoid") and regularization term (searching from $0.01,0.1,1,10,100$ ) were tuned, and finally the "linear" kernel and 1 of $C$ were selected. As for stacking, LR and SVM were served as the base classifiers in the first layer, and LR was used for the final prediction in the second layer.

Given that the imbalanced classes between cognitive trajectories, synthetic minority oversampling technique (SMOTE) [28] was used to process the training data before prediction. In the stage of performance evaluation, balanced accuracy, considering both positive and negative classes, were chosen to measure how accurate is the overall performance of prediction model is. Also, the F1 score, which combines both precision and recall, was also calculated. Area under the receiver operating characteristic curve (AUROC) and its 95\% confidence interval $(95 \% \mathrm{Cl})$ were used to evaluate the discrimination of prediction models, and calibration was evaluated by brier score. In order to obtain stable estimation of model performance, we looped through the code 1000 times for LR and 100 times for SVM and stacking (for time consideration).

\section{Sensitivity analysis}

Given that trajectory analysis is more stable for participants with 3 or more observations over time, we conducted a sensitivity analyses by including participants who had complete information on MMSE for at least 3 waves. Followed by the selection criteria in this study, 1668 participants were included for sensitivity analysis. Besides, trajectory of cognitive function may also be influenced by potential factors such as age, sex, and education [29]. Therefore, a further sensitivity analysis was performed with a consideration of age, sex, and education as covariates for participants who had complete information on MMSE for at least 2 waves $(n=3502)$.

\section{Statistical analysis}

Continuous variables were presented as mean \pm standard deviation. Categorical variables were presented as percentages. The comparisons of baseline characteristics among different trajectories were performed by appropriately choosing ANOVA test and chi square test. All the above analysis were conducted with SPSS 25.0. Trajectory class analyses were performed with Mplus 8.3 (Muthén and Muthén, 2019). Feature selection, model derivation, and model evaluation were performed with scikit-learn package in Python 3.7.6. A two-sided $p$-value of $<0.05$ was considered statistically significant.

\section{Results}

\section{Heterogeneous trajectories of cognitive function}

A linear model was chosen for LCGM model because a relatively lower SABIC was observed in a linear LGCM model. Among the 1 to 5-class LCGM models, a 2-class model were selected because of higher entropy $(0.92)$, and a $>5 \%$ smallest class ( $p$ value of VLMR-LRT was $<0.0001)$. Finally, a better fit (SABIC 
$=48305.86$, entropy $=0.93, p$ value of VLMR-LRT $<0.0001)$ and good separation of different classes (smallest class $=6.40 \%$ ) were also observed in the GMM model.

Table 1

Performance of latent class growth model and growth mixture model

\begin{tabular}{|lllll|}
\hline & SABIC & Entropy & VLMR-LRT & Smallest class \\
\hline LGCM & & & & \\
\hline linear & 49352.73 & - & - & - \\
\hline quadratic & 49359.34 & - & - & - \\
\hline cubic & 49361.33 & - & - & \\
\hline LCGM & & & & $100 \%$ \\
\hline 1-class & 49804.05 & - & - & $7.17 \%$ \\
\hline 2-class & 48356.83 & 0.92 & 0.0000 & $4.91 \%$ \\
\hline 3-class & 47668.92 & 0.91 & 0.1883 & $1.31 \%$ \\
\hline 4-class & 47201.83 & 0.89 & 0.4556 & $1.37 \%$ \\
\hline 5-class & 46879.68 & 0.84 & 0.6411 & $6.40 \%$ \\
\hline GMM & 48305.86 & 0.93 & & 0.0082 \\
\hline 2-class & & & & \\
\hline $\begin{array}{l}\text { NOTE: SABIC, sample-size adjusted Bayesian information criteria; VLMR-LRT, VUONG-LO-MENDELL- } \\
\text { RUBIN likelihood ratio test; LGCM, latent growth curve model; LCGM, latent class growth model; GMM, } \\
\text { growth mixture model. }\end{array}$ & & & \\
\hline
\end{tabular}

Figure 1 shows the mean MMSE scores over time for the two trajectory classes: stable-function (93.6\%, intercept $=28.402, p<0.001$; slope $=-0.138, p<0.001)$, and rapid-decline $(6.4 \%$, intercept $=24.410, p<$ 0.001 ; slope $=-0.2 .454, p<0.005)$. The sensitivity analyses identified cognitive trajectories with similar shapes and distributions among participants who had complete information on MMSE for at least 3 waves (Supplementary Figure S1). With adjusting age, sex, and education, similar results were also observed among participants who had complete information on MMSE for at least 2 waves (Supplementary Figure S2), and we found that there were differences in the baseline MMSE score between different genders (higher in man compared with woman, $p<0.001$, Supplementary Table S2) as well as between different education level (higher in high education level compared with low education level, $p<0.001)$, but the effects of sex, and education on the slope were not significant $(p=0.227, p=$ 0.394 , respectively). Meanwhile, age showed a negative effect on the MMSE score in each wave (all $p$ values $<0.001)$. 
Baseline characteristics of study population 
Table 2

Sample Characteristics by cognitive trajectories classes in 2002

\begin{tabular}{lllll}
$\begin{array}{l}\text { Total sample }(\mathrm{N} \\
=3502)\end{array}$ & \multicolumn{2}{l}{ Trajectory Class } & P-value\# & $\begin{array}{l}\text { Missing } \\
\text { data }\end{array}$ \\
\cline { 2 - 3 } & Normal & $\begin{array}{l}\text { Rapid- } \\
\text { decline }\end{array}$ & \\
& $(n=$ & & \\
$3278)$ & $(n=224)$ & &
\end{tabular}

\section{Sociodemographic Characteristics}

$\begin{array}{lllll}\text { Age } & 75.64 \pm 8.17 & 75.23 \pm & 81.62 \pm & <0.001\end{array}$

Sex

\begin{tabular}{|c|c|c|c|c|}
\hline Male & $2108(60.2 \%)$ & $\begin{array}{l}2012 \\
(61.4 \%)\end{array}$ & $\begin{array}{l}96 \\
(42.9 \%)\end{array}$ & $<0.001$ \\
\hline Female & $1394(39.8 \%)$ & $\begin{array}{l}1266 \\
(38.6 \%)\end{array}$ & $\begin{array}{l}128 \\
(57.1 \%)\end{array}$ & \\
\hline
\end{tabular}

Ethnicity

Han Ancestry

$3335(95.2 \%)$

3113

$(95.0 \%)$

222

$(99.1 \%)$

0.005

$167(4.8 \%)$

165

$(5.0 \%)$

$2(0.9 \%)$

Minority

Education

Illiterate

$1280(36.6 \%)$

1144

$(35.0 \%)$

136

$(60.7 \%)$

$<0.001$

6

$\begin{array}{lll}1226(35.1 \%) & 1163 & 63 \\ & (35.5 \%) & (28.1 \%)\end{array}$

Junior High and above

$990(28.3 \%)$

965

$(29.5 \%) \quad(11.2 \%)$

Marital Status

Unmarried

$39(1.1 \%)$

$37(1.1 \%)$

$2(0.9 \%)$

$<0.001$

Married

2020 (57.7\%)

1943

77

(59.3\%) (34.4\%)

NOTE. Values are presented as mean \pm standard deviation, number (\%), or median (interquartile range).

* PWB = psychological well-being, $A D L=$ activity of daily living, IADL = instrumental activity of daily living.

\# Null hypothesis: no difference across the two classes 


\begin{tabular}{|c|c|c|c|c|c|}
\hline & \multirow{2}{*}{$\begin{array}{l}\text { Total sample }(\mathrm{N} \\
=3502)\end{array}$} & \multicolumn{2}{|c|}{ Trajectory Class } & \multirow[t]{2}{*}{$P$-value\# } & \multirow{2}{*}{$\begin{array}{l}\text { Missing } \\
\text { data }\end{array}$} \\
\hline & & $\begin{array}{l}\text { Normal } \\
(\mathrm{n}= \\
3278)\end{array}$ & $\begin{array}{l}\begin{array}{l}\text { Rapid- } \\
\text { decline }\end{array} \\
(n=224)\end{array}$ & & \\
\hline Separated/Divorced/Widowed & $1443(41.2 \%)$ & $\begin{array}{l}1298 \\
(39.6 \%)\end{array}$ & $\begin{array}{l}145 \\
(64.7 \%)\end{array}$ & & \\
\hline \multicolumn{6}{|l|}{ Residence } \\
\hline Urban & $1652(47.2 \%)$ & $\begin{array}{l}1567 \\
(47.8 \%)\end{array}$ & $\begin{array}{l}85 \\
(37.9 \%)\end{array}$ & \multirow[t]{2}{*}{0.004} & \multirow[t]{2}{*}{-} \\
\hline Rural & $1850(52.8 \%)$ & $\begin{array}{l}1711 \\
(52.2 \%)\end{array}$ & $\begin{array}{l}139 \\
(62.1 \%)\end{array}$ & & \\
\hline \multicolumn{6}{|l|}{ Co-residence } \\
\hline Yes & 3015 (86.1\%) & $\begin{array}{l}2838 \\
(86.6 \%)\end{array}$ & $\begin{array}{l}177 \\
(79.0 \%)\end{array}$ & \multirow[t]{2}{*}{0.002} & \multirow[t]{2}{*}{-} \\
\hline No & $487(13.9 \%)$ & $\begin{array}{l}440 \\
(13.4 \%)\end{array}$ & $\begin{array}{l}47 \\
(21.0 \%)\end{array}$ & & \\
\hline \multicolumn{6}{|l|}{ Lifestyles } \\
\hline \multicolumn{6}{|l|}{ Regular Fruit Intake } \\
\hline Yes & $1400(40.0 \%)$ & $\begin{array}{l}1328 \\
(40.5 \%)\end{array}$ & $\begin{array}{l}72 \\
(32.1 \%)\end{array}$ & \multirow[t]{2}{*}{0.013} & \multirow[t]{2}{*}{-} \\
\hline No & $2102(60.0 \%)$ & $\begin{array}{l}1950 \\
(59.5 \%)\end{array}$ & $\begin{array}{l}152 \\
(67.9 \%)\end{array}$ & & \\
\hline \multicolumn{6}{|l|}{ Regular Vegetables Intake } \\
\hline Yes & $3184(90.9 \%)$ & $\begin{array}{l}2987 \\
(91.1 \%)\end{array}$ & $\begin{array}{l}197 \\
(87.9 \%)\end{array}$ & \multirow[t]{2}{*}{0.109} & \multirow[t]{2}{*}{-} \\
\hline No & $318(9.1 \%)$ & $\begin{array}{l}291 \\
(8.9 \%)\end{array}$ & $\begin{array}{l}27 \\
(12.1 \%)\end{array}$ & & \\
\hline \multicolumn{6}{|l|}{ Regular Tea Consumption } \\
\hline Yes & 1331 (38.0\%) & $\begin{array}{l}1267 \\
(38.7 \%)\end{array}$ & $\begin{array}{l}64 \\
(28.6 \%)\end{array}$ & 0.003 & - \\
\hline \multicolumn{6}{|c|}{$\begin{array}{l}\text { NOTE. Values are presented as mean } \pm \text { standard deviation, number (\%), or median (interquartile } \\
\text { range). }\end{array}$} \\
\hline \multicolumn{6}{|c|}{$\begin{array}{l}\text { *PWB = psychological well-being, } A D L=\text { activity of daily living, } I A D L=\text { instrumental activity of daily } \\
\text { living. }\end{array}$} \\
\hline
\end{tabular}




\begin{tabular}{|c|c|c|c|c|c|}
\hline & \multirow{2}{*}{$\begin{array}{l}\text { Total sample }(\mathrm{N} \\
=3502)\end{array}$} & \multicolumn{2}{|c|}{ Trajectory Class } & \multirow[t]{2}{*}{$P$-value\# } & \multirow{2}{*}{$\begin{array}{l}\text { Missing } \\
\text { data }\end{array}$} \\
\hline & & $\begin{array}{l}\text { Normal } \\
(n= \\
3278)\end{array}$ & $\begin{array}{l}\begin{array}{l}\text { Rapid- } \\
\text { decline }\end{array} \\
(n=224)\end{array}$ & & \\
\hline No & $2171(62.0 \%)$ & $\begin{array}{l}2011 \\
(61.3 \%)\end{array}$ & $\begin{array}{l}160 \\
(71.4 \%)\end{array}$ & & \\
\hline \multicolumn{6}{|l|}{ Smoker } \\
\hline Yes & $954(27.2 \%)$ & $\begin{array}{l}907 \\
(27.7 \%)\end{array}$ & $\begin{array}{l}47 \\
(21.0 \%)\end{array}$ & \multirow[t]{2}{*}{0.029} & \multirow[t]{2}{*}{$\begin{array}{l}1 \\
(0.03 \%)\end{array}$} \\
\hline No & $2547(72.8 \%)$ & $\begin{array}{l}2370 \\
(72.3 \%)\end{array}$ & $\begin{array}{l}177 \\
(79.0 \%)\end{array}$ & & \\
\hline \multicolumn{6}{|l|}{ Alcohol Drinker } \\
\hline Yes & $953(27.2 \%)$ & $\begin{array}{l}897 \\
(27.4 \%)\end{array}$ & $\begin{array}{l}56 \\
(25.1 \%)\end{array}$ & \multirow[t]{2}{*}{0.461} & \multirow[t]{2}{*}{$\begin{array}{l}3 \\
(0.09 \%)\end{array}$} \\
\hline No & $2546(72.8 \%)$ & $\begin{array}{l}2379 \\
(72.6 \%)\end{array}$ & $\begin{array}{l}167 \\
(74.9 \%)\end{array}$ & & \\
\hline \multicolumn{6}{|l|}{ Regular Exercise } \\
\hline Yes & $1682(48.1 \%)$ & $\begin{array}{l}1592 \\
(48.6 \%)\end{array}$ & $\begin{array}{l}90 \\
(40.4 \%)\end{array}$ & \multirow[t]{2}{*}{0.017} & \multirow[t]{2}{*}{$\begin{array}{l}2 \\
(0.06 \%)\end{array}$} \\
\hline No & $1818(51.9 \%)$ & $\begin{array}{l}1685 \\
(51.4 \%)\end{array}$ & $\begin{array}{l}133 \\
(59.6 \%)\end{array}$ & & \\
\hline \multicolumn{6}{|l|}{ Leisure Activity } \\
\hline Low Frequency & $2016(57.6 \%)$ & $\begin{array}{l}1835 \\
(56.0 \%)\end{array}$ & $\begin{array}{l}181 \\
(80.8 \%)\end{array}$ & \multirow[t]{2}{*}{$<0.001$} & \multirow[t]{2}{*}{-} \\
\hline High Frequency & $1486(42.4 \%)$ & $\begin{array}{l}1443 \\
(44.0 \%)\end{array}$ & $\begin{array}{l}43 \\
(19.2 \%)\end{array}$ & & \\
\hline \multicolumn{6}{|c|}{ Mental State \& Metal State } \\
\hline PWB Score* & $27.25 \pm 4.10$ & $\begin{array}{l}27.29 \pm \\
4.10\end{array}$ & $\begin{array}{l}26.76 \pm \\
3.96\end{array}$ & 0.062 & - \\
\hline ADL Score* & $11.88 \pm 0.56$ & $\begin{array}{l}11.90 \pm \\
0.52\end{array}$ & $\begin{array}{l}11.61 \pm \\
0.97\end{array}$ & $<0.001$ & - \\
\hline
\end{tabular}

NOTE. Values are presented as mean \pm standard deviation, number (\%), or median (interquartile range).

* $P W B=$ psychological well-being, $A D L=$ activity of daily living, $I A D L=$ instrumental activity of daily living.

\# Null hypothesis: no difference across the two classes 


\begin{tabular}{|c|c|c|c|c|c|}
\hline & \multirow{2}{*}{$\begin{array}{l}\text { Total sample }(\mathrm{N} \\
=3502)\end{array}$} & \multicolumn{2}{|c|}{ Trajectory Class } & \multirow[t]{2}{*}{$P$-value\# } & \multirow{2}{*}{$\begin{array}{l}\text { Missing } \\
\text { data }\end{array}$} \\
\hline & & $\begin{array}{l}\text { Normal } \\
(n= \\
3278)\end{array}$ & $\begin{array}{l}\text { Rapid- } \\
\text { decline } \\
(n=224)\end{array}$ & & \\
\hline IADL Score* & $14.74 \pm 2.66$ & $\begin{array}{l}14.85 \pm \\
2.53\end{array}$ & $\begin{array}{l}13.16 \pm \\
3.86\end{array}$ & $<0.001$ & - \\
\hline \multicolumn{6}{|c|}{ Chronic Disease } \\
\hline \multicolumn{6}{|l|}{ Hypertension } \\
\hline Yes & $625(18.4 \%)$ & $\begin{array}{l}589 \\
(18.5 \%)\end{array}$ & $\begin{array}{l}36 \\
(16.8 \%)\end{array}$ & \multirow[t]{2}{*}{0.546} & \multirow[t]{2}{*}{$\begin{array}{l}100 \\
(2.86 \%)\end{array}$} \\
\hline No & $2777(81.6 \%)$ & $\begin{array}{l}2599 \\
(81.5 \%)\end{array}$ & $\begin{array}{l}178 \\
(83.2 \%)\end{array}$ & & \\
\hline \multicolumn{6}{|l|}{ Diabetes } \\
\hline Yes & $85(2.5 \%)$ & $80(2.5 \%)$ & $5(2.3 \%)$ & \multirow[t]{2}{*}{0.875} & \multirow{2}{*}{$\begin{array}{l}100 \\
(2.86 \%)\end{array}$} \\
\hline No & $3317(97.5 \%)$ & $\begin{array}{l}3108 \\
(97.5 \%)\end{array}$ & $\begin{array}{l}209 \\
(97.7 \%)\end{array}$ & & \\
\hline \multicolumn{6}{|l|}{ Stroke } \\
\hline Yes & $168(4.9 \%)$ & $\begin{array}{l}158 \\
(5.0 \%)\end{array}$ & $10(4.7 \%)$ & \multirow[t]{2}{*}{0.878} & \multirow[t]{2}{*}{$\begin{array}{l}100 \\
(2.86 \%)\end{array}$} \\
\hline No & $3234(95.1 \%)$ & $\begin{array}{l}3032 \\
(95.0 \%)\end{array}$ & $\begin{array}{l}202 \\
(95.3 \%)\end{array}$ & & \\
\hline \multicolumn{6}{|c|}{$\begin{array}{l}\text { NOTE. Values are presented as mean } \pm \text { standard deviation, number }(\%) \text {, or median (interquartile } \\
\text { range). }\end{array}$} \\
\hline \multicolumn{6}{|c|}{$\begin{array}{l}\text { * PWB = psychological well-being, } A D L=\text { activity of daily living, } I A D L=\text { instrumental activity of daily } \\
\text { living. }\end{array}$} \\
\hline \multicolumn{6}{|c|}{ \# Null hypothesis: no difference across the two classes } \\
\hline
\end{tabular}

Table 2 shows the results of baseline characteristics of the study sample with different trajectory classes. This study identified 3278 participants with "stable-function" class, 224 participants with "rapid-decline" class. The mean age of the whole population was 75.64 years; and $60.2 \%$ of them were male. Of the whole population, $36.6 \%$ were illiterate, $57.5 \%$ were in married, and $52.5 \%$ of them were rural residents. Most of them were Han ancestry (95.2\%) and lived with their household members (86.1\%). About $91.0 \%$ participants often had vegetables intake, while a much lower proportion were existed in fresh fruit intake $(40.0 \%)$, tea consumption (38.0\%), regular exercise (48.1\%), and high frequency leisure activity $(42.4 \%)$. The smoker and alcohol drinker accounted both $27.2 \%$. The baseline mental state score was 27.25 , and the baseline ADL and IADL scores were 11.88, and 14.74, respectively. Hypertension (18.4\%) was 
common among the older, followed by stroke (4.9\%) and diabetes (2.5\%). For the comparisons of baseline characteristics among trajectory classes, significant differences were found in all variables except for alcohol drinker, mental state score, and chronic diseases (hypertension, diabetes, and stroke). Comparisons between the analytical sample and drop-out sample are presented in Supplementary Tables S3. The sample in the current analysis was younger, having higher education level, having higher frequency leisure activity, better mental state, better cognitive functioning, better activity of daily living, having diabetes, and more likely to be rural participants.

\section{Determinants of cognitive trajectories}

Table 3 summarizes the results of the associations between potential risk factors and cognitive trajectories. Compared with the stable-function class, individuals who were older, being female, being Han ancestry, having low level education, living in rural districts, having low-frequency leisure activity, having low ADL score were more likely to develop a rapid-decline trajectory. 
Table 3

Binary logistic regression analysis for influencing factors of cognitive trajectories

\begin{tabular}{|c|c|c|}
\hline & Odds Ratio (95\% Cl) & $P$-value \\
\hline Age & $1.07(1.05-1.09)$ & 0.000 \\
\hline \multicolumn{3}{|l|}{ Sex (Ref: Male) } \\
\hline Female & $1.70(1.17-2.48)$ & 0.005 \\
\hline \multicolumn{3}{|l|}{ Ethnicity (Ref: Han Ancestry) } \\
\hline Minority & $0.17(0.04-0.69)$ & 0.013 \\
\hline \multicolumn{3}{|l|}{ Education (Ref: Illiterate) } \\
\hline Primary School & $0.65(0.45-0.93)$ & 0.018 \\
\hline Junior High and above & $0.40(0.24-0.67)$ & 0.000 \\
\hline \multicolumn{3}{|l|}{ Marital Status (Ref: Unmarried) } \\
\hline Married & $1.15(0.26-5.13)$ & 0.855 \\
\hline Divorced/Separated/Widowed & $0.85(0.19-3.88)$ & 0.833 \\
\hline \multicolumn{3}{|l|}{ Residence (Ref: Rural) } \\
\hline Urban & $0.72(0.51-1.00)$ & 0.049 \\
\hline \multicolumn{3}{|l|}{ Co-residence (Ref: No) } \\
\hline Yes & $0.91(0.61-1.36)$ & 0.646 \\
\hline \multicolumn{3}{|l|}{ Regular Fruit Intake (Ref: No) } \\
\hline Yes & $0.81(0.58-1.15)$ & 0.238 \\
\hline \multicolumn{3}{|c|}{ Regular Vegetables Intake (Ref: No) } \\
\hline Yes & $0.93(0.58-1.50)$ & 0.758 \\
\hline \multicolumn{3}{|c|}{ Regular Tea Consumption (Ref: No) } \\
\hline Yes & $0.79(0.57-1.10)$ & 0.165 \\
\hline \multicolumn{3}{|l|}{ Smoker (Ref: No) } \\
\hline Yes & $1.00(0.67-1.47)$ & 0.978 \\
\hline
\end{tabular}

Note: reference is the stable-function class.

* $P W B=$ psychological well-being, $A D L=$ activity of daily living, $I A D L=$ instrumental activity of daily living. 


\begin{tabular}{|llc|}
\hline & Odds Ratio (95\% Cl) & P-value \\
\hline Yes & $1.22(0.85-1.77)$ & 0.285 \\
\hline Regular Exercise (Ref: No) & $0.97(0.71-1.34)$ & \\
Yes & & 0.871 \\
\hline Leisure Activity (Ref: Low Frequency) & $0.58(0.39-0.84)$ & 0.005 \\
\hline High Frequency & $1.03(0.99-1.07)$ & 0.169 \\
\hline PWB Score* & $0.75(0.61-0.91)$ & 0.005 \\
\hline ADL Score* & $0.99(0.94-1.04)$ & 0.643 \\
\hline IADL Score* & & 0.617 \\
\hline Hypertension (Ref: No) & $0.90(0.60-1.35)$ & \\
\hline Yes & & 0.463 \\
\hline Diabetes (Ref: No) & & \\
\hline Yes & & 0.538 \\
\hline Stroke (Ref: No) & & \\
\hline Yes & & \\
\hline Note: reference is the stable-function class. & & \\
\hline * PWB = psychological well-being, ADL = activity of daily living, IADL = instrumental activity of daily & \\
\hline living. & & \\
\hline
\end{tabular}

\section{Performance evaluation of cognitive trajectory prediction models}

The performance of cognitive trajectory prediction models with the whole variables and selected variables are shown in Table 4. Generally, all the three ML algorithms performed relatively better in the feature selection data set compared with the full variables data set. Specifically, LR, SVM, and stacking had equivalent performance, with a balanced accuracy around 0.66 , a highest sensitivity of 0.74 in SVM, a specificity of 0.58 , a F1 score of 0.73 in both LR and SVM. For AUROC, the three algorithms achieved $0.66,0.66$, and 0.65 , respectively. Also, the three models calibrated well with their brier scores ranged $0.25-0.26$. The sensitivity analysis also showed similar performance for predicting "stable-function" vs "rapid-decline" classes among participants who had complete information on MMSE for at least 3 waves (Supplementary Table S4) as well as two-classes trajectories considering covariates among participants who had complete information on MMSE for at least 2 waves (Supplementary Table S5). 
Table 4

Performance of machine learning algorithms for cognitive trajectory prediction.

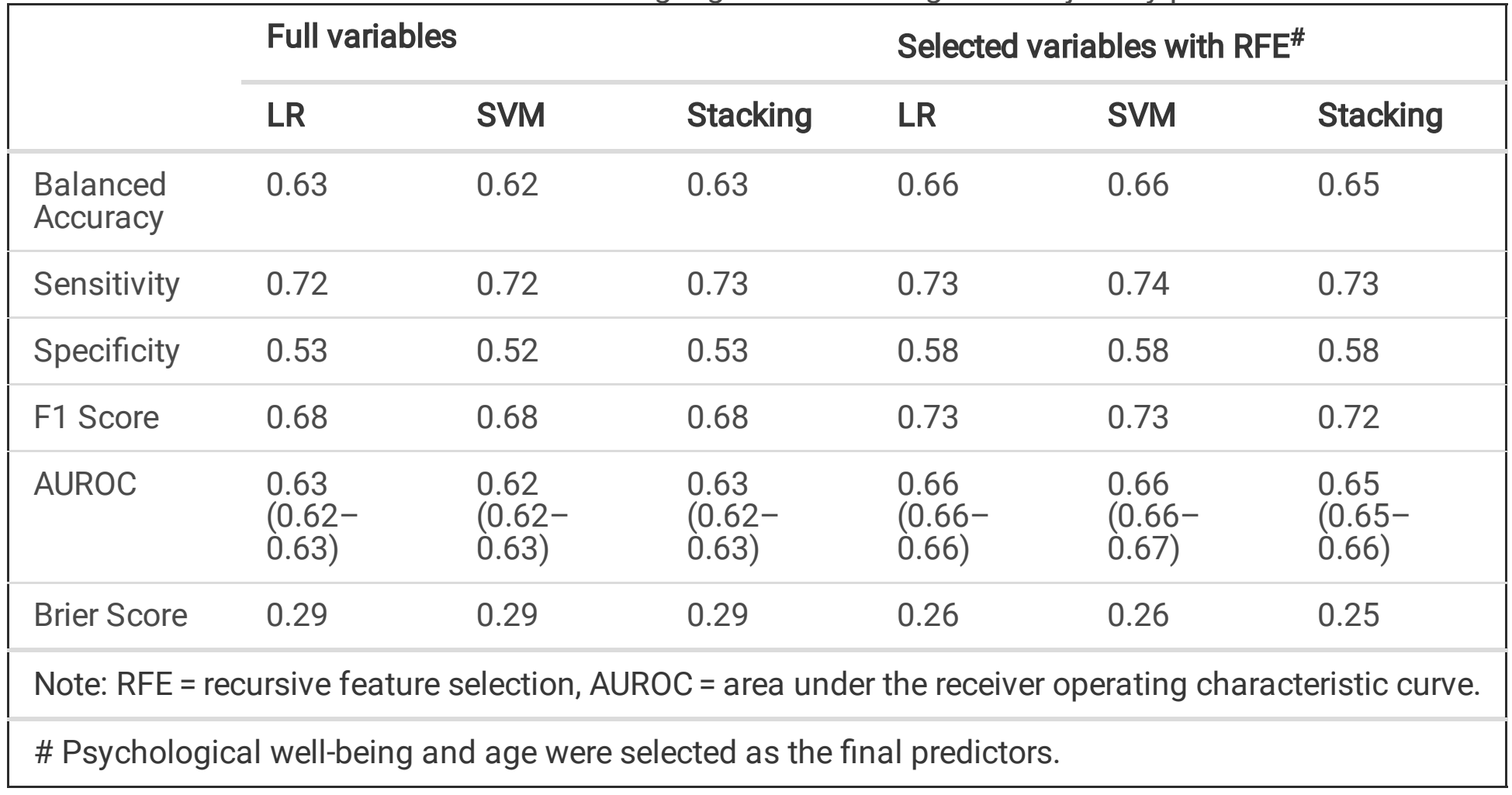

\section{Discussion}

In the current study, we described the cognitive trajectories of 16-year follow-up in a nationally representative sample of community-dwelling older Chinese and explored its potential determinants. The results indicated that a two-classes linear model of cognitive trajectories provided the best statistical fit measures, namely, stable-function, and rapid-decline. Previous studies have examined for cognitive trajectories and have reported varying numbers of trajectories, Brent J.Small et al. identified a two-classes trajectories with growth mixed model over a six year period [30]. Similarly, the two-classes cognitive trajectories (stable and decline) were also reported in the Alzheimer's Disease Neuroimaging Initiative (ADNI) study at 9 timepoints spanned over 6 years [11]. While 6 trajectories were identified for modeling cognitive and psychotic symptoms among Alzheimer patients over a 13.5 years follow-up [31]. The 2classes model were more popular in studies such as distinguishing AD converter vs. non-converter, and stable vs. decline $[11,32,33]$. The results revealed that stable-function class accounted for most of the population [5, 34], indicating that majority of participants kept stable cognitive function through their aging periods.

Compared with stable-function class, individuals with older age, female gender, Han ethnicity, having no schooling, rural residents, low frequency leisure activities, and low baseline ADL score were at much higher risk of having rapid cognitive decline, which were also demonstrated in previous studies [29, 3538]. The human aging process, a significant risk factor of cognitive impairment, has been demonstrated to be closely associated with cognition decline. A hypothesis, derived from that hormone-replacement therapy can protect against dementia, suggested that the changing of hormone may result in sex 
difference in cognition decline [39]. High level education is one of important protective factors of cognitive performance, people can get high education by implementing public policy to support highquality education in early life. What's more, setting up universities for the elderly to promote education, encouraging the elderly to strengthen exercise and social interaction also means a lot. There exist huge differences between rural and urban. Rural residents in mainland China usually have lower income, live in poorer housing conditions, receive less education, and enjoy lower levels of social and health entitlements compared with the urban ones [40]. Also, for elderly, mild cognition impairment is easy to be overlooked and it is difficult to distinguish cognitive impairment form normal cognition decline with aging. More seriously, in rural areas, due to limited utilization of healthcare resources, the screening or diagnosis of cognitive impairment in rural areas might be delayed and underestimated [41]. Recently, government have made great progression to reduce the rural-urban inequality and focus on the modification of associated factors. It suggested that absence of leisure activities can be viewed as the base of cognition decline. One possible explanation is that the leisure activities can increase the cognitive stimuli and promote the cognitive function. Positive correlation between leisure activities and brain volume, white matter, as well as parietal lobe gray matter volume in older adults has been established through a magnetic resonance imaging study [42]. On the other hand, leisure activities can promote interpersonal social contact among elderly, which is also known to be associated with a decreased risk of cognitive impairment [43]. An increasing amount of scientific evidence suggested that some leisure activities, such as tai chi, can reduce perceived and physiological stress and improves cognition in healthy older adults [44]. Besides, reading, playing mahjong or cards are common in elderly, they played positive role in delaying cognitive decline and promote healthy aging. Previous study showed that ADL, physical performance, and cognitive impairment prevalence gradually increased with aging [45]. ADL is closely affected by medical care and life nursing, indicating the demand of medical care investment and long-term care.

A few studies have focused on the prediction of cognitive-related trajectories, but mainly for diagnostic conversion such as $\mathrm{MCl}$ converts into $\mathrm{AD}[9,46]$. Due to the differences in task definitions and data types, it is not trivial to compare the performance of prediction models. For instance, Brent J. Small et al. found that a $67 \%$ of sensitivity and $86 \%$ of specificity were obtained for GMM classification of two decline trajectories [30]. Nikhil Bhagwat et al. used a ML framework to predict the clinical symptom trajectories among Alzheimer patients, and achieved with 0.90 accuracy and 0.97 AUROC for two-classes trajectories task and 0.76 accuracy for 3-classes task on ADNI datasets, as well as 0.72 accuracy and 0.88 AUROC for two-classes task on replication in another dataset [11]. In the current study, we found that ML algorithms had potential to predict the trajectory of cognitive function with acceptable performance, especially for its relatively higher sensitivity (0.74), F1 score (0.73), and low brier score (0.25). However, the performance of prediction models in current study was not particularly high, of which limited prediction data might be an explanation, and more complicated information such as imaging data was deserved for future study. Considering the fact that the severity of cognitive decline and limited early screening tool, thus, our prediction models might be meaningful for the primary screening of high-risk populations. Also, data used in our prediction models were easy to obtain with low costs and no injury 
risk, so it was suitable for early warning in the community level or less developed areas. Certainly, the current prediction task was an attempt for the risk identification of cognitive trajectories served as the preliminary task before clinical diagnosis and treatment, and also help facilitate the widespread use of machine learning algorithms for health risk prediction.

In the current study, age and psychological well-being (PWB) were identified as the important predictors. Previous studies have found that age was the independent risk factor of cognition impairment and PWB is verified as significant predictors to mild cognitive impairment, and PWB should not be overlooked in intervention trials $[47,48]$. Thus, family caregivers and medical staff should pay more attention to the potential changes in the cognitive state of older adults as well as their psychological well-being. Engaging in volunteering, provision of informal care, looking after grandchildren, paid employment may help to promote psychological well-being in some extent, besides some policy strategies, such as facilitating welfare benefits, improving the service capacity and level of public hospitals, satisfying the need for some health-care services should also be considered.

There may be some potential advantages in the current study. Firstly, the cognitive trajectory classes were examined by GMM model using a 16-year longitudinal data, which was helpful for uncovering the heterogeneity of long-term changing patterns of cognitive function among older adults. Besides, ML algorithms incorporated easily accessible epidemiological information would be target for early screening of high-risk populations, and promote healthy cognitive development.

\section{Limitations}

However, the shortcomings of present study should be noted. Firstly, the sample size was relatively small and types of predictors were limited, while machine learning was superior in prediction tasks with large amount of data and multi-source information, especially for the current mainstream brain imaging tests. This might be an important reason for the limited performance in the current prediction task. However, brain imaging tests were relatively expensive, which was also an obstacle for widely use in early risk identification. So, simple tools with high performance based on easy-accessible information need to be designed in future studies. Secondly, data in 2002 was used to predict the 16-year span trajectories, and all predictors were assumed to be unchangeable, but in fact, some variables might change over time. Therefore, the power of predictors would be weakened due to a long-term follow-up. For a further analysis, more advanced machine learning methods, such as long short-term memory (LSTM) model with the ability for analyzing time series data [49], could be used for prediction of cognitive trajectories. Finally, the MMSE was used for cognitive measurement in current study. The advantage of the MMSE is that it is a well-known instrument and provides an easy-to-interpret score reflecting global cognition. However, a main disadvantage of the scale is its limited sensitivity to change over time and the presence of ceiling effects, especially in cognitively unimpaired populations. While for the current study, we just focused on the changing patterns of MMSE score rather than distinguishing among $\mathrm{MCl} v \mathrm{vs}$. $\mathrm{AD}$, so this may not be a main challenge in study. Perhaps more sensitive measure such as delayed recall or a functional measure would be much valuable for identifying trajectories, and it may deserve more attention in future studies. 


\section{Conclusions}

This study identified two trajectories of cognitive function among older adults in China using a community-based, nationally representative sample. Older age, female gender, Han ethnicity, having no schooling, rural residents, low frequency leisure activities, and low baseline ADL score were associated with the rapid decline in cognitive function. Also, machine learning algorithms showed potential for classifying different trajectories, serving as a tool for early risk identification. In conclusion, understanding the heterogeneity of cognitive function and its determinants would be helpful for promotion of cognitive function among older adults.

\section{Abbreviations}

MMSE: Mini-mental State Examination; ML: machine learning; LR: logistic regression; SVM: support vector machine; AUROC: area under the receiver operating characteristic curve; RFE: recursive feature elimination; LGCM: latent growth curve model; LCGM: latent class growth model; GMM: growth mixed model; PWB: psychological well-being; ADL: activity of daily living; IADL: instrumental activity of daily living.

\section{Declarations}

\section{Ethical Approval and Consent to participate}

The CLHLS study was approved by the Research Ethics Committee of Peking University (IRB0000105213074), and written informed consent was provided by all participants or their proxy respondents.

\section{Consent for publication}

Not applicable.

\section{Availability of data and materials}

The dataset supporting the conclusions of this article is publicly available at the Peking University Open Research Data (https://opendata.pku.edu.cn/dataverse/CHADS).

\section{Competing interests}

None.

\section{Funding}

This study was supported by the National Natural Science Foundation of China (No. 81973144). 
Y.W., M.J., C.X., S.L., Z.J. and Y.F. worked together on this article. Specifically, Y.W. conceived and designed the study. Y.W., Z.J. and C.X. contributed to the data analysis. Y.W., M.J., and C.X. drafted the manuscript. S.L. revised the article. Y.F. supervised and revised the article. All authors have read and approved the final manuscript.

\section{Acknowledgements}

We thank the staff and the participants of the CLHLS study.

\section{References}

1. Zhao L. 2020 Alzheimer's disease facts and figures. Alzheimers Dement 2020, 16(3):391-460.

2. Lane CA, Hardy J, Schott JM. Alzheimer's disease. Eur J Neurol 2018, 25(1):59-70.

3. Min JW. A longitudinal study of cognitive trajectories and its factors for Koreans aged 60 and over: A latent growth mixture model. Int J Geriatr Psych 2018, 33(5):755-762.

4. Taniguchi Y, Kitamura A, Murayama H, Amano H, Shinozaki T, Yokota I et al. Mini-Mental State Examination score trajectories and incident disabling dementia among community-dwelling older Japanese adults. Geriatr Gerontol Int 2017, 17(11):1928-1935.

5. Tu LH, Lv XZ, Yuan CZ, Zhang M, Fan ZL, Xu XL et al. Trajectories of cognitive function and their determinants in older people: 12 years of follow-up in the Chinese Longitudinal Healthy Longevity Survey. Int Psychogeriatr 2020, 32(6):765-775.

6. Olaya B, Bobak M, Haro JM, Demakakos P. Trajectories of Verbal Episodic Memory in Middle-Aged and Older Adults: Evidence from the English Longitudinal Study of Ageing. J Am Geriatr Soc 2017, 65(6):1274-1281.

7. Licher S, Leening MJG, Yilmaz P, Wolters FJ, Heeringa J, Bindels PJE et al. Development and Validation of a Dementia Risk Prediction Model in the General Population: An Analysis of Three Longitudinal Studies. Am J Psychiat 2019, 176(7):543-551.

8. Prestia A, Caroli A, Wade SK, van der Flier WM, Ossenkoppele R, Van Berckel B et al. Prediction of AD dementia by biomarkers following the NIA-AA and IWG diagnostic criteria in $\mathrm{MCI}$ patients from three European memory clinics. Alzheimers Dement 2015, 11(10):1191-1201.

9. Davatzikos C, Bhatt P, Shaw LM, Batmanghelich KN, Trojanowski JQ. Prediction of MCI to AD conversion, via MRI, CSF biomarkers, and pattern classification. Neurobiol Aging 2011, 32(12).

10. Ahn HJ, Chin J, Park A, Lee BH, Suh MK, Seo SW et al. Seoul Neuropsychological Screening BatteryDementia Version (SNSB-D): A Useful Tool for Assessing and Monitoring Cognitive Impairments in Dementia Patients. J Korean Med Sci 2010, 25(7):1071-1076.

11. Bhagwat N, Viviano JD, Voineskos AN, Chakravarty MM, Neuroimaging AsD. Modeling and prediction of clinical symptom trajectories in Alzheimer's disease using longitudinal data. Plos Comput Biol 2018, 14(9). 
12. Lee JS, Cho SK, Kim HJ, Kim YJ, Park KC, Lockhart SN et al. Prediction Models of Cognitive Trajectories in Patients with Nonamnestic Mild Cognitive Impairment. Sci Rep-Uk 2018, 8.

13. Nevin L, Editors PM. Advancing the beneficial use of machine learning in health care and medicine: Toward a community understanding. Plos Med 2018, 15(11).

14. Zhang R, Simon G, Yu F. Advancing Alzheimer's research: A review of big data promises. Int J Med Inform 2017, 106:48-56.

15. Tanveer M, Richhariya B, Khan RU, Rashid AH, Khanna P, Prasad M et al. Machine Learning Techniques for the Diagnosis of Alzheimer's Disease: A Review. Acm T Multim Comput 2020, 16(1).

16. Lv YB, Gao X, Yin ZX, Chen HS, Luo JS, Brasher MS et al. Revisiting the association of blood pressure with mortality in oldest old people in China: community based, longitudinal prospective study. BmjBrit Med J 2018, 361.

17. Zeng Y, Feng QS, Hesketh T, Christensen K, Vaupel JW. Survival, disabilities in activities of daily living, and physical and cognitive functioning among the oldest-old in China: a cohort study. Lancet 2017, 389(10079):1619-1629.

18. Yi Z. Reliability of Age Reporting Among the Chinese Oldest-Old in the CLHLS Datasets: Springer Netherlands; 2008.

19. An RP, Liu GG. Cognitive impairment and mortality among the oldest-old Chinese. Int J Geriatr Psych 2016, 31(12):1345-1353.

20. Zhang W, Liu G. Childlessness, Psychological Well-being, and Life Satisfaction Among the Elderly in China. Journal of cross-cultural gerontology 2007, 22(2).

21. Wu Z, Schimmele CM. Psychological disposition and self-reported health among the 'oldest-old' in China. Ageing Soc 2006, 26:135-151.

22. Han WJ, Shibusawa T. Trajectory of physical health, cognitive status, and psychological well-being among Chinese elderly. Arch Gerontol Geriat 2015, 60(1):168-177.

23. Xiao J, Shi Z, Fang Y. Association Between Disability Trajectory and Health Care Service Utilization Among Older Adults in China. Journal of the American Medical Directors Association 2021.

24. Wu XY, Yuan XL, Wang W, Liu K, Qin Y, Sun XL et al. Value of a Machine Learning Approach for Predicting Clinical Outcomes in Young Patients With Hypertension. Hypertension 2020, 75(5):12711278.

25. Qiu PY, Zeng M, Kuang WH, Meng SS, Cai Y, Wang HL et al. Heterogeneity in the dynamic change of cognitive function among older Chinese people: A growth mixture model. Int J Geriatr Psych 2020, 35(10):1123-1133.

26. Liu SW, Rovine MJ, Molenaar PCM. Selecting a Linear Mixed Model for Longitudinal Data: Repeated Measures Analysis of Variance, Covariance Pattern Model, and Growth Curve Approaches. Psychol Methods 2012, 17(1):15-30.

27. Wolpert DH. Stacked Generalization. Neural Networks 1992, 5(2):241-259. 
28. Chawla NV, Bowyer KW, Hall LO, Kegelmeyer WP. SMOTE: Synthetic minority over-sampling technique. J Artif Intell Res 2002, 16:321-357.

29. Wu Z, Phyo AZZ, Al-Harbi T, Woods RL, Ryan J. Distinct Cognitive Trajectories in Late Life and Associated Predictors and Outcomes: A Systematic Review. J Alzheimers Dis Rep 2020, 4(1):459478.

30. Small BJ, Backman L. Longitudinal trajectories of cognitive change in preclinical Alzheimer's disease: A growth mixture modeling analysis. Cortex 2007, 43(7):826-834.

31. Wilkosz PA, Seltman HJ, Devlin B, Weamer EA, Lopez OL, DeKosky ST et al. Trajectories of cognitive decline in Alzheimer's disease. Int Psychogeriatr 2010, 22(2):281-290.

32. Huang MY, Yang W, Feng QJ, Chen WF, Neuroimaging AsD. Longitudinal measurement and hierarchical classification framework for the prediction of Alzheimer's disease. Sci Rep-Uk 2017, 7.

33. Moradi E, Pepe A, Gaser C, Huttunen H, Tohka J, Initi AsDN. Machine learning framework for early MRI-based Alzheimer's conversion prediction in MCl subjects. Neuroimage 2015, 104:398-412.

34. Hayden KM, Reed BR, Manly JJ, Tommet D, Pietrzak RH, Chelune GJ et al. Cognitive decline in the elderly: an analysis of population heterogeneity. Age Ageing 2011, 40(6):684-689.

35. Legdeur N, Heymans MW, Comijs HC, Huisman M, Maier AB, Visser PJ. Age dependency of risk factors for cognitive decline. Bmc Geriatr 2018, 18.

36. Yates LA, Ziser S, Spector A, Orrell M. Cognitive leisure activities and future risk of cognitive impairment and dementia: systematic review and meta-analysis. Int Psychogeriatr 2016, 28(11):1791-1806.

37. Zhu XY, Qiu CX, Zeng Y, Li J. Leisure activities, education, and cognitive impairment in Chinese older adults: a population-based longitudinal study. Int Psychogeriatr 2017, 29(5):727-739.

38. Downer B, Chen NW, Raji M, Markides KS. A longitudinal study of cognitive trajectories in Mexican Americans age 75 and older. Int J Geriatr Psych 2017, 32(10):1122-1130.

39. LeBlanc ES, Janowsky J, Chan BKS, Nelson HD. Hormone replacement therapy and cognition Systematic review and meta-analysis. Jama-J Am Med Assoc 2001, 285(11):1489-1499.

40. Lu Y, Liu C, Yu D, Fawkes S, Ma J, Zhang M et al. Prevalence of mild cognitive impairment in community-dwelling Chinese populations aged over 55 years: a meta-analysis and systematic review. Bmc Geriatr 2021, 21(1):10.

41. Liu CC, Liu CH, Sun Y, Lee HJ, Tang LY, Chiu MJ. Rural-urban disparities in the prevalence of mild cognitive impairment and dementia in Taiwan: A door-to-door nationwide study. J Epidemio/2021.

42. Benedict C, Brooks SJ, Kullberg J, Nordenskjold R, Burgos J, Le Greves M et al. Association between physical activity and brain health in older adults. Neurobiol Aging 2013, 34(1):83-90.

43. Marioni RE, Proust-Lima C, Amieva H, Brayne C, Matthews FE, Dartigues JF et al. Social activity, cognitive decline and dementia risk: a 20-year prospective cohort study. Bmc Public Health 2015, 15.

44. Wu MT, Tang PF, Goh JOS, Chou TL, Chang YK, Hsu YC et al. Task-Switching Performance Improvements After Tai Chi Chuan Training Are Associated With Greater Prefrontal Activation in 
Older Adults. Front Aging Neurosci 2018, 10.

45. Zhang PD, Lv YB, Li ZH, Yin ZX, Li FR, Wang JN et al. Age, Period, and Cohort Effects on Activities of Daily Living, Physical Performance, and Cognitive Functioning Impairment Among the Oldest-Old in China. J Gerontol a-Biol 2020, 75(6):1214-1221.

46. Ye JP, Farnum M, Yang E, Verbeeck R, Lobanov V, Raghavan N et al. Sparse learning and stability selection for predicting MCI to AD conversion using baseline ADNI data. Bmc Neurol 2012, 12.

47. Chen LL. Leisure activities and psychological wellbeing reduce the risk of cognitive impairment among older adults with hearing difficulty: A longitudinal study in China. Maturitas 2021, 148:7-13.

48. Gates N, Valenzuela M, Sachdev PS, Singh MAF. Psychological well-being in individuals with mild cognitive impairment. Clin Interv Aging 2014, 9:779-792.

49. Hong X, Lin RJ, Yang CH, Zeng NY, Cai CT, Gou J et al. Predicting Alzheimer's Disease Using LSTM. leee Access 2019, 7:80893-80901.

\section{Figures}




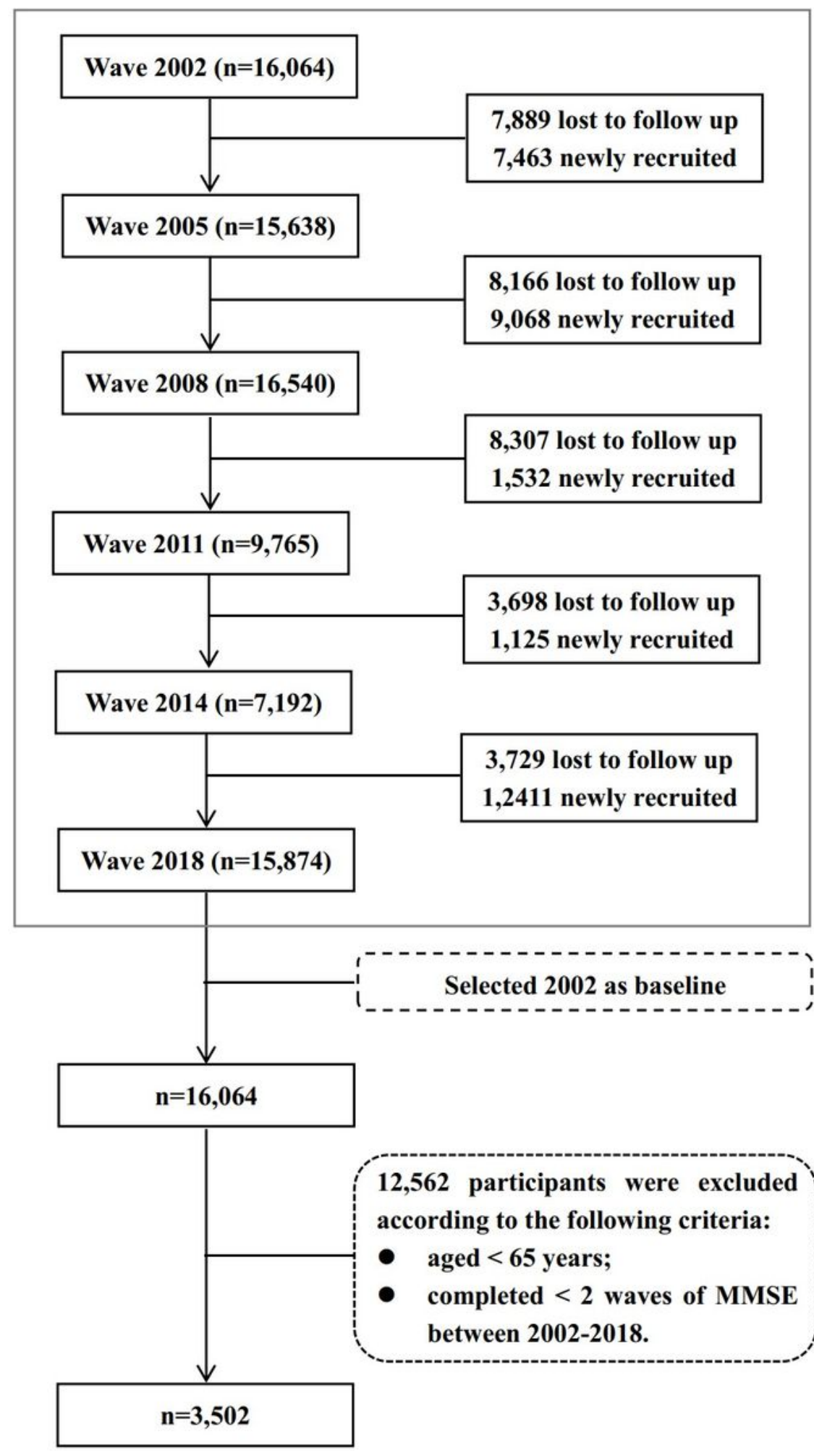

Figure 1

Flow chart of CLHLS survey and sample selection in current analysis. 


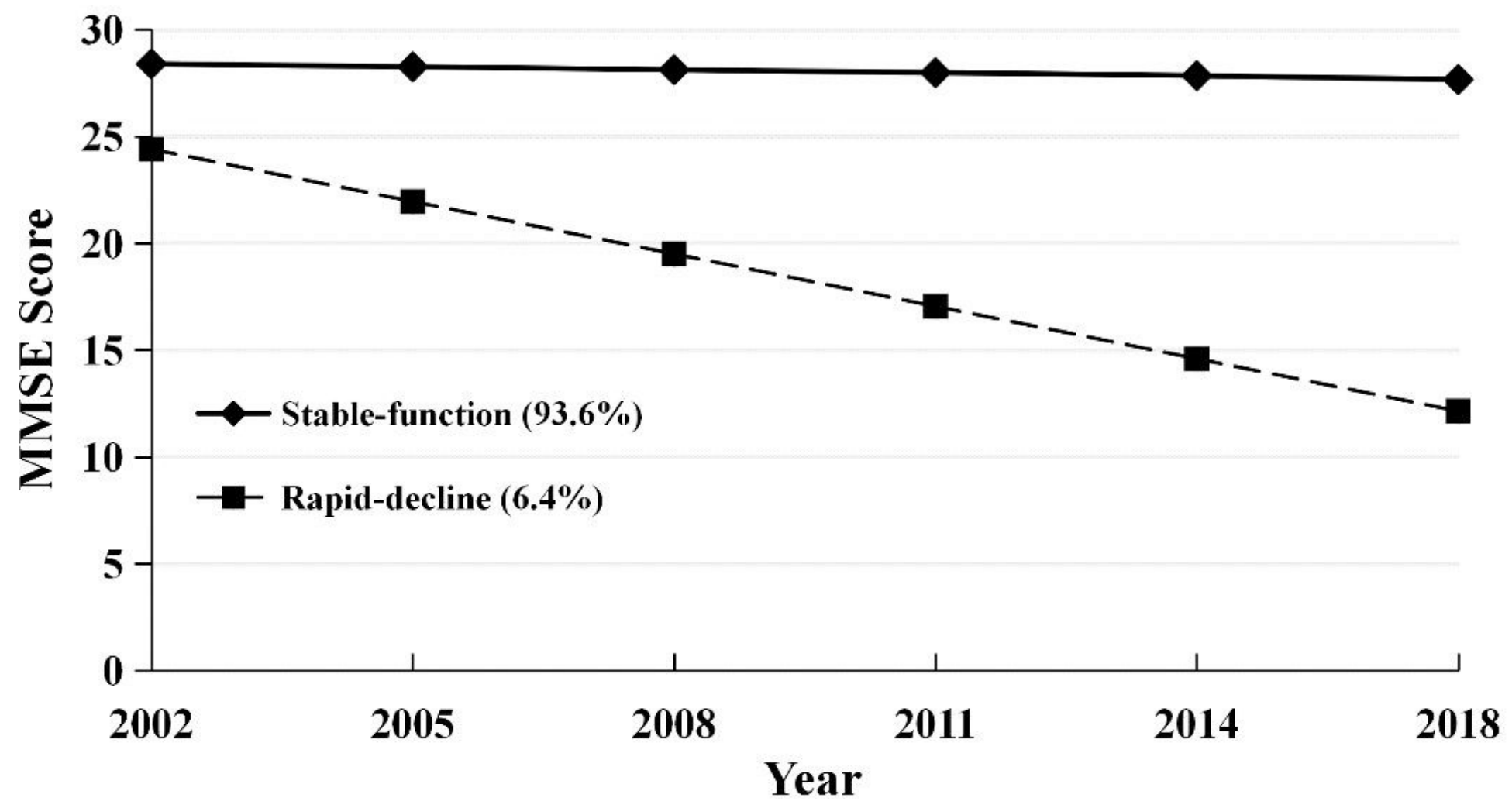

Figure 2

Heterogeneous trajectories of cognitive function. MMSE scores were measured by the Chinese version Mini-Mental State Examination. Two classes were identified: "stable-function" class, and "rapid-decline" class. Each trajectory was the mean change pattern of MMSE over time.

\section{Supplementary Files}

This is a list of supplementary files associated with this preprint. Click to download.

- SupplementaryInformation.docx 